\title{
MEST promotes bladder cancer cell proliferation, migration and invasion via STAT3/Twist-1-mediated EMT
}

\author{
Cheng Zhao, Xiheng Hu, Shiyu Tong, Miao Mo, Wei He, Long Wang, Yangle Li \\ Department of Urology, Xiangya Hospital, Central South University, Changsha, China \\ Contributions: (I) Conception and design: Y Li, C Zhao; (II) Administrative support: L Wang; (III) Provision of study materials or patients: C Zhao, X \\ Hu, S Tong; (IV) Collection and assembly of data: M Mo, W He; (V) Data analysis and interpretation: L Wang; (VI) Manuscript writing: All authors; \\ (VII) Final approval of manuscript: All authors. \\ Correspondence to: Yangle Li. Department of Urology, Xiangya Hospital, Central South University, No 87 Xiangya Road, Changsha 410008, China. \\ Email: liyangle2003@163.com.
}

Background: Mesoderm-specific transcript (MEST) has been demonstrated to be a proto-oncogene or anti-oncogene in various carcinomas. However, the role and mechanism of MEST in bladder cancer (BC) are still unknown. Here we aimed to explore the effect of MEST on malignant biological behaviour in BC and its potential mechanism.

Methods: The expression of MEST in BC tissues and cells was detected by qRT-PCR methods. MEST depletion and overexpression cell lines were established in T24 and 5637 respectively. Then the effects of MEST on cell proliferation, migration, invasion and epithelial-mesenchymal transition (EMT) were investigated. Finally, the STAT3/Twist-1 signaling was verified.

Results: MEST was elevated in BC tissues and cells lines, and its high expression was highly relevant to the clinicopathologic features of patients with BC and to poor prognosis in these patients. MEST depletion impeded cell proliferation, migration and invasion as well as epithelial-mesenchymal transition (EMT), while MEST overexpression promoted malignant biological behaviour in BC. Mechanistically, MEST upregulated p-STAT3 and Twist-1 expression, while treatment with a STAT3 inhibitor clearly attenuated the STAT3 activation and Twist-1 upregulation induced by MEST. Subsequently, rescue assays confirmed that inhibition of STAT3 signalling could remarkably relieve the oncogenic effects of MEST on malignant biological behaviour in BC.

Conclusions: Our data confirmed that MEST exerts oncogenic functions in bladder cancer via STAT3/ Twist-1 signalling and that MEST may represent a promising target in BC treatment.

Keywords: Mesoderm-specific transcript (MEST); epithelial-mesenchymal transition; STAT3/Twist-1 signalling; bladder cancer (BC)

Submitted Feb 13, 2020. Accepted for publication Sep 17, 2020.

doi: $10.21037 /$ tcr-20-1006

View this article at: http://dx.doi.org/10.21037/tcr-20-1006

\section{Introduction}

Bladder cancer (BC) is a common human urinary malignancy and is ranked as the ninth most common cancer worldwide. Each year, more than 4,000,000 cases of BC are diagnosed and over 160,000 die from the disease worldwide (1). Unfortunately, the morbidity and mortality rates are also increasing, and although the current treatments for BC have substantially improved, over $50 \%$ of patients develop local progression and relapse after surgery $(2,3)$. The prognosis of patients with bladder cancer, especially those at an advanced stage, is still unsatisfactory (4). Due to its high morbidity and postoperative recurrence, the exploration and clarification of the molecular mechanisms of BC progression are crucial, as this knowledge may contribute to potential therapeutic targets and improvements in the 
therapeutic effect.

Mesoderm-specific transcript (MEST) is an imprinted gene located at the human $7 \mathrm{q} 32.2$ locus, and since it is preferentially expressed on the paternal allele, it is also called paternal expressed gene 1 (PEG1) (5). At present, the biological function of MEST is unclear, but previous studies have demonstrated that MEST is involved in the growth, differentiation and maintenance of mesoderm cells and contributes to embryonic development $(6,7)$. Recent research has indicated that MEST frequently undergoes loss of imprinting (LOI) in several malignancies, such as thyroid carcinoma (8), lung cancer $(9,10)$ breast cancer (11) uterine leiomyoma (12) and colorectal cancer (13), which may be linked to MEST upregulation and cancer progression. MEST has also been reported to be a tumour suppressor in ovarian cancer (14), which suggests it can suppress cell proliferation, migration, invasion, angiogenesis and tumorigenesis, while in thyroid carcinoma (8) and breast cancer (15), MEST has been described as an oncogene, and therefore contributes to cell proliferation and metastasis. However, little is known about the role of MEST in BC.

In this study, we demonstrated that MEST was elevated in $\mathrm{BC}$ tissues and cell lines and that high MEST expression was closely related to the clinicopathologic features of BC patients as well as poor prognosis in these patients. We also found that MEST can facilitate cell proliferation, migration and invasion via STAT3/Twist-1-mediated EMT.

We present the following article in accordance with the MDAR checklist (available at http://dx.doi.org/10.21037/ tcr-20-1006).

\section{Methods}

\section{Clinic bladder cancer samples}

Bladder cancer tissues $(\mathrm{n}=68)$ and adjacent normal tissues $(n=20)$ were collected from Xiangya hospital, Central South University, from Jan 2018 to Dec 2018. The adjacent tissues were obtained from at least $5 \mathrm{~cm}$ away from tumor border and with no microscopic tumor cells. No patient was administered to preoperative chemotherapy and radiotherapy. All the tissues were fast frozen in liquid nitrogen and kept at $-80^{\circ} \mathrm{C}$. The study was conducted in accordance with the Declaration of Helsinki (as revised in 2013). The study was approved by the institutional ethics committee of Xiangya Hospital, Central South University (approval No. 2019012618), and written informed consents were obtained from all patients. The pathological features were acquired from patients' medical records.

\section{Cell culture and transfection}

Human bladder cancer cell lines 5637, UM-UC-3, T24 and bladder epithelial immortalized cell line SV-HUC-1 were obtained from COBIOER (Nanjing, China) and cultured following to the instructions.

siRNAs were purchased from GenePharm (Shanghai, China). siRNA knockdown was carried out with a siRNA pool which includes 2 siRNAs targeting the MEST cDNA sequence. The sequences information as showing in Table 1 . Scramble oligonucleotides were used as a negative control. MEST cDNA ORF plasmid was purchased from Sino Biological (Beijing, China). Cells were transfected with Lipofectamine 3000 (Thermo Fisher, USA) following the manufacturers' instructions. Cells were collected at $48 \mathrm{~h}$ after transfection.

\section{$q R T-P C R$}

Total RNAs were isolated using Trizol (Sigma-Aldrich, USA). ExcelRT ${ }^{\mathrm{TM}}$ Kit (Gene, Hongkong, Chia) was applied to RNA reverse transcription according to the product's protocol. The SYBR Green I (TOYOBO, Japan) was used to conduct the qRT-PCR reaction on the LightCycler480 system (Roche, Germany). $\beta$-actin was utilised as a control for normalizing. Each reaction was performed in triplicate. The specific primers for amplifying each gene were listed in Table 1.

\section{Western blot}

Protein was extracted from cells with RIPA lysis containing protease inhibitors and phosphate inhibitors. Equal amounts proteins $(30 \mu \mathrm{g} / \mathrm{samples})$ were separated by $10 \%$ SDSPAGE gel and immunoblotted onto a polyvinylidene difluoride (PVDF) membrane (ThermoFisher, USA). After blocking with $5 \%$ non-fat dry milk, the membrane was incubated with primary antibodies against MEST (1:500, Proteintech), E-cadherin (1:500, Proteintech), N-cadherin (1:800, Proteintech), Vimentin (1:8000, Proteintech), STAT3 (1:1000, Abcam), p-STAT3 (Try705) (1:1000, Abcam), Twist-1 (1:500, Proteintech), overnight at $4{ }^{\circ} \mathrm{C}$. After washing by PBS, the membrane was covered with HRP-conjugated secondary antibody, the immune signals were examined using enhanced chemiluminescence reagent (Thermofisher, USA). GAPDH employed as the loading control. 
Table 1 The sequences of siRNAs and qRT-PCR primers

\begin{tabular}{ll}
\hline Name & \multicolumn{1}{c}{ Sequence } \\
\hline siRNAs & GGAGGUAUCUUUCCUGAGA \\
MEST siRNA-1 & CAACAAUGACGGGAACUUA \\
MEST siRNA-2 & UUCUCCGAACGUGUCACGU \\
scramble siRNA & \\
qRT-PCR & TGTGGGTGTGGTTGGAAGTC \\
MEST forward & CCTCAAGGTCAGACCCTTCC \\
MEST reverse & TTCTGCTGCTCTTGCTGTTT \\
E-cadherin forward & TGGCTCAAGTCAAAGTCCTG \\
E-cadherin reverse & CAAGATGGGTCAATGGAAATAG \\
N-cadherin forward & CTCAGGAATACGAGCCTTCAC \\
N-cadherin reverse & CCAGGCAAAGCAGGAGTC \\
Vimentin forward & GGGTATCAACCAGAGGGAGT \\
Vimentin reverse & TTCCTTCCTGGGCATGGAGTC \\
$\beta$-actin forward & TCTTCATTGTGCTGGGTGCC \\
\hline siRNAs, small interfering RNAs; qRT-PCR, quantitative real-time \\
PCR.
\end{tabular}

\section{MTT and clone formation analysis}

Cell viability and proliferation were investigated by MTT and clone formation experiments respectively. For MTT, each group cells $\left(5 \times 10^{4}\right.$ cells/well $)$ were plated into 96 -well plates and cultured at $37^{\circ} \mathrm{C}$ for $24 \mathrm{~h}$. Then, each well was added $20 \mu \mathrm{L}$ MTT and incubated for $4 \mathrm{~h}$. After removing the medium, each well was added $150 \mu \mathrm{L}$ of DMSO and incubated at $37{ }^{\circ} \mathrm{C}$ for $10 \mathrm{~min}$. Finally, the absorbance was read at $570 \mathrm{~nm}$ by the microplate reader (Multishan Go, Bio-Tek, USA). For clone formation assay, $5 \times 10^{3}$ cells were plated into the $35 \mathrm{~mm}$ dish and cultured for two weeks. After that, colonies of cells were fixed in $4 \%$ paraformaldehyde (PFA) and stained with $0.01 \%$ crystal violet dye.

\section{Cell migration and invasion analysis}

Cell migration was analyzed by wound scratch experiment. Simply, cells were plated onto 12 -well plates and grew close to $100 \%$ confluence. Cell wounds were scratched using a $20 \mu \mathrm{L}$ pipette tube. Wound closure was measured by the distance between the opposite edges of the wound after
0 and $24 \mathrm{~h}$. Cell invasion was detected with the BioCoat Matrigel Invasion chamber (Corning, USA). $5 \times 10^{5}$ cells suspended in serum-free medium were implanted into the upper chambers (coated with matrigel), while the lower chambers were added medium containing $10 \%$ FBS. Incubating at $37{ }^{\circ} \mathrm{C}$ for $48 \mathrm{~h}$, the translocated cells were fixed with $4 \% \mathrm{PFA}$ and stained by $0.1 \%$ crystal violet for $20 \mathrm{~min}$. Subsequently, the invasive cells were captured and counted under the microscope.

\section{Immunofluorescence}

Cells $\left(5 \times 10^{3}\right)$ were implanted in a 24 -well plate paved with sterile slips. $24 \mathrm{~h}$ later, the cells were fixed in PFA for $0.5 \mathrm{~h}$ and permeabilized in $0.1 \%$ Triton $\mathrm{X}-100$ for $5 \mathrm{~min}$. Then, the slips were incubated with anti-E-cadherin (20874-1AP, Proteintch, USA) or Vimentin (10366-1AP, Proteintch, USA) antibody at a dilution of 1:100 overnight at $4{ }^{\circ} \mathrm{C}$. Subsequently, the slips were covered by fluorescently labeled secondary antibody for 2 hours. Then, DAPI was used to stain nuclei. Finally, fluorescence was observed and captured under the fluorescent microscope (ECLIPSE NI, Nikon, Japan).

\section{Statistical analysis}

Each experiment was performed at least three times, and the data were presented by mean \pm standard deviation (SD). The statistical analysis was conducted by SPSS 20.0 software (SPSS Inc., IL, USA). Student's $t$-test was applied to analyse the differential expression between the two groups. A chisquare test was applied to assess the relationship between MEST expression and clinicopathological features of BC. $\mathrm{P}<0.05$ were identified statistically significant.

\section{Results}

\section{MEST was upregulated in BC tissues and its upregulation was associated poor survival}

Firstly, MEST expression was investigated in 68 BC and 20 adjacent normal tissues by qRT-PCR assay, we observed that MEST was upregulated in BC tissues (Figure 1A). Moreover, MEST expression in lymph node (LN)-metastatic BC tissues was remarkably higher than nonmetastatic tumor tissues (Figure 1B). Basing on the median value of MEST expression in BC tissues, the patients were divided into the low expression group 
A

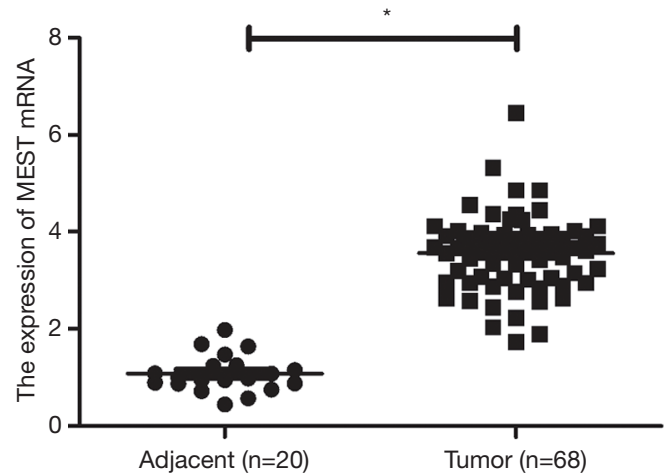

C

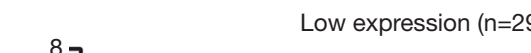

B

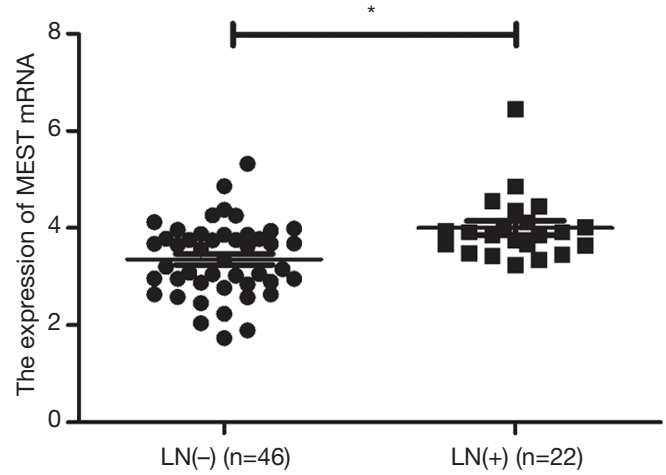

High expression $(n=39)$
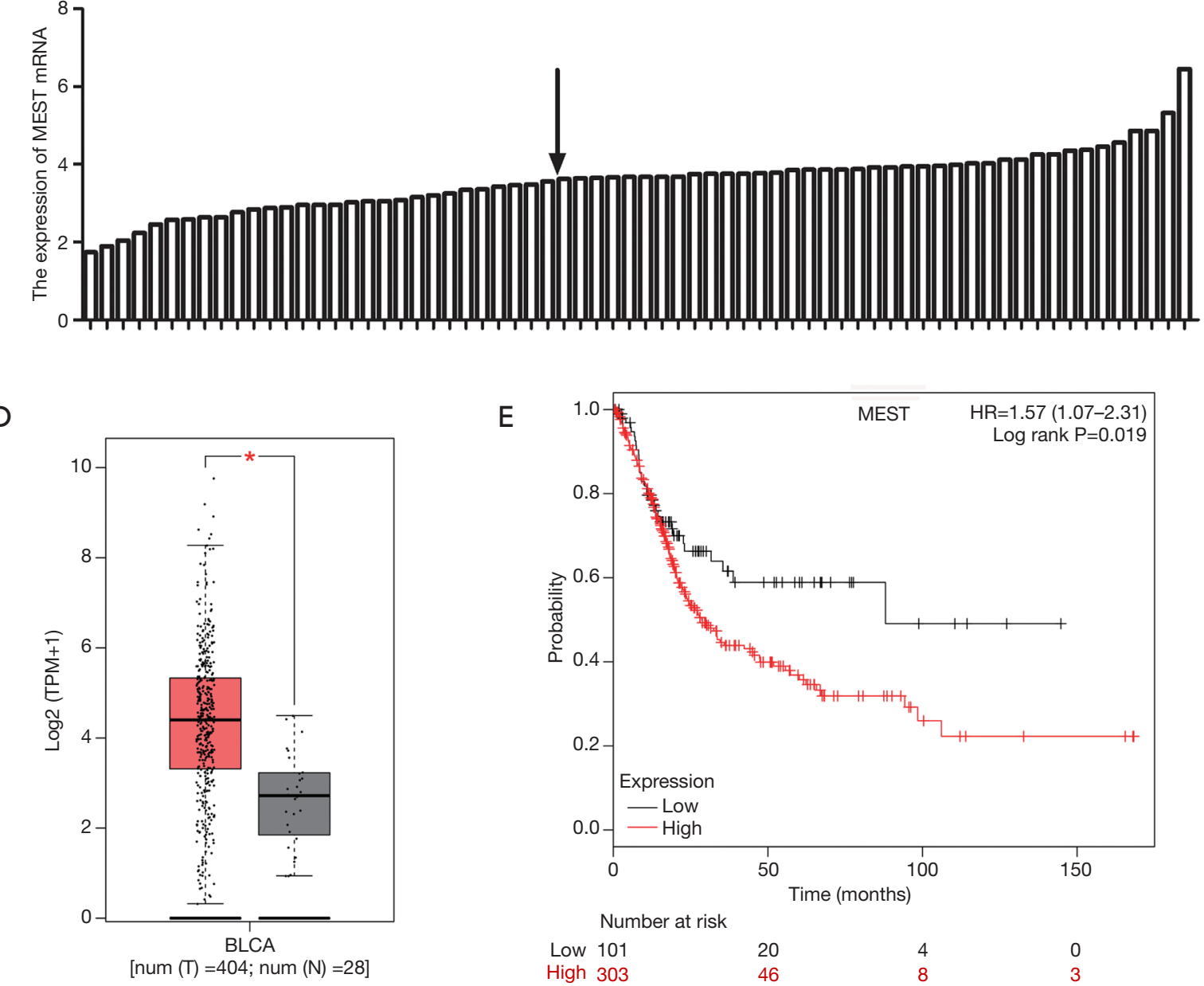

Figure 1 MEST was upregulated in bladder cancer (BC) tissues and its upregulation was associated poor survival. (A) Quantitative Realtime PCR (qRT-PCR) detected MEST expression in 68 BC and 20 adjacent normal tissues; (B) Expression of MEST was detected in lymph node (LN) metastatic BC [LN(+)] and nonmetastatic tumor tissues [LN(-)]; (C) BC patients were divided into high expression and low expression groups according to the median value of MEST expression; (D) The expression of MEST was analyzed in BC tissues from Gene Expression Profiling Interactive Analysis (GEPIA) data (http://gepia.cancer-pku.cn). (E) The overall survival of BC patients was evaluated (analyzed using Kaplan-Meier Plotter, http://kmplot.com/analysis/). ${ }^{*} \mathrm{P}<0.05$. 
Table 2 Association between MEST expression and clinicopathologic characteristics in bladder cancer

\begin{tabular}{|c|c|c|c|c|}
\hline Features & Number of cases & \multicolumn{2}{|c|}{ MEST expression } & $\chi^{2}$ test, $\mathrm{P}$ value \\
\hline Age (years) & & & & 0.462 \\
\hline$\geq 60$ & 38 & 18 & 20 & \\
\hline$<60$ & 30 & 11 & 19 & \\
\hline Female & 42 & 17 & 25 & \\
\hline Male & 26 & 12 & 14 & \\
\hline Tumor stage & & & & $0.014^{*}$ \\
\hline $\mathrm{T} 1+\mathrm{T} 2$ & 28 & 17 & 11 & \\
\hline Low & 39 & 12 & 27 & \\
\hline High & 29 & 17 & 12 & \\
\hline LN metastasis & & & & $0.035^{\star}$ \\
\hline $\mathrm{LN}(+)$ & 22 & 5 & 17 & \\
\hline $\mathrm{LN}(-)$ & 46 & 24 & 22 & \\
\hline
\end{tabular}

$\mathrm{LN}(+)$, lymph node $(\mathrm{LN})$ metastasis; $\mathrm{LN}(-)$, no lymph node metastasis. ${ }^{*} \mathrm{P}<0.05$.

$(\mathrm{n}=29)$ and the high expression group (n=39) (Figure 1C). The correlation between MEST expression and BC clinicopathological features was analyzed by $\chi^{2}$ test. We found that MEST expression was obviously correlated with tumor stage $(\mathrm{P}=0.014)$, grade $(\mathrm{P}=0.027)$ and $\mathrm{LN}$ metastasis ( $\mathrm{P}=0.035)$ (Table 2). Besides, GEPIA database (http://gepia. cancer-pku.cn) showed that MEST was elevated in BC tissues (Figure 1D). Additionally, basing on the TCGA data analysis, the high MEST expression was indicated the poor overall survival for BCpatients (Figure 1E).

\section{MEST promoted BC cell proliferation, migration, invasion}

To explore the function of MEST in BC cells, Firstly, we assessed its endogenous level in BC cell lines (5637, UM-UC-3, T24) and bladder epithelial cell SV-HUC-1 with Western blot. The result showed that MEST was obviously upregulated in tumor cell lines compare to SVHUC-1 cells, Moreover, T24 cells had a higher expression than 5637 and UM-UC-3 cells (Figure 2A). Therefore, we depleted MEST level in T24 cells and overexpressed MEST in 5637 cells. Western blot detection demonstrated that MEST expression was effectively reduced in T24 cells and increased in 5637 cells respectively (Figure 2B). MTT and clone formation assays showed that MEST knockdown obviously suppressed T24 cell proliferation and growth, while overexpression of MEST in 5637 cells had a remarkable enhancing effect (Figure $2 C$ and $D$ ). Wound scratch and invasion assays demonstrated that MEST depletion strongly impaired cell migration and invasion, but the result was the opposite when over-expression MEST in 5637 cells (Figure $2 E$ and $F$ ).

\section{MEST induced epithelial-mesenchymal transition (EMT) in $B C$}

Considering EMT as one crucial process in tumor invasion and metastasis, the effect of MEST on EMT was investigated by examining the level of EMT markers, such as the epithelial marker E-cadherin and mesenchymal markers N-cadherin and Vimentin. qRT-PCR and western blot assays demonstrated that MEST knockdown obviously represses the expression of mesenchymal markers $\mathrm{N}$-cadherin and Vimentin, and upregulated 
A
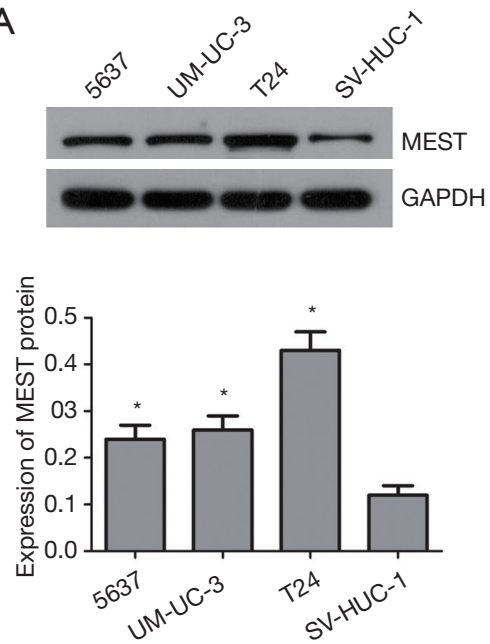

D

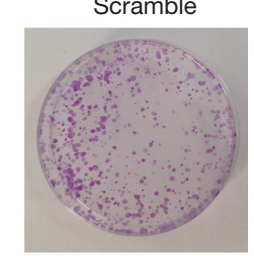

Vector

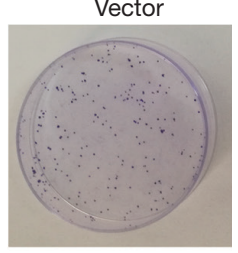

E
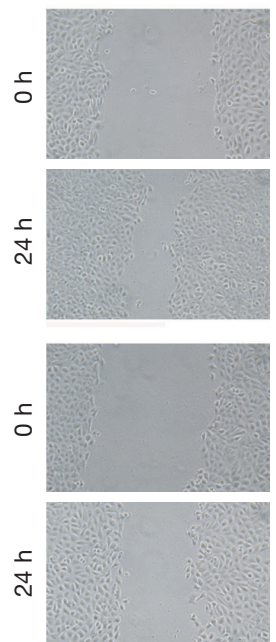

MEST
B
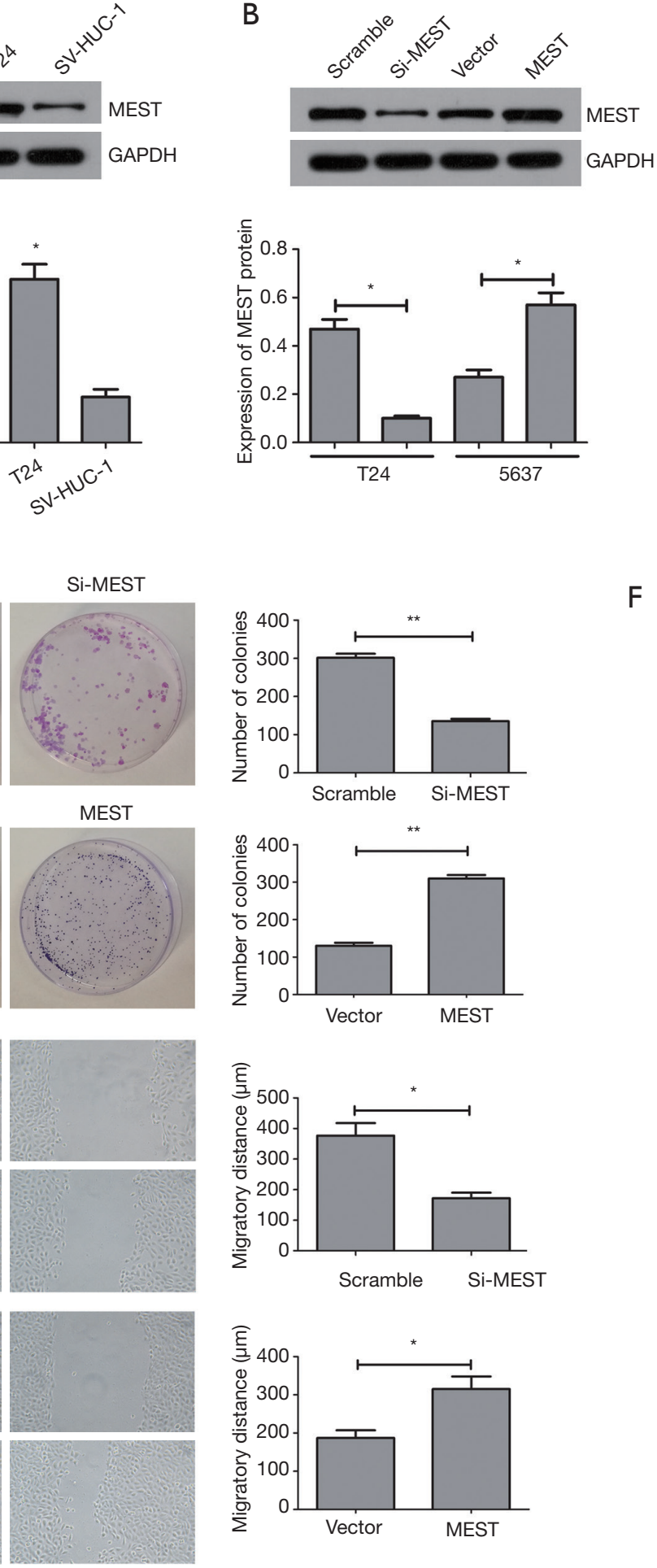
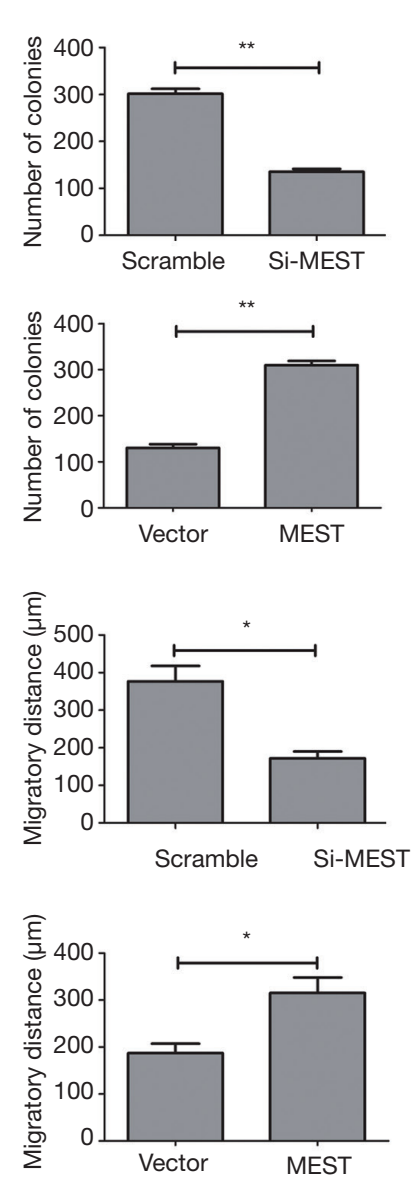

C
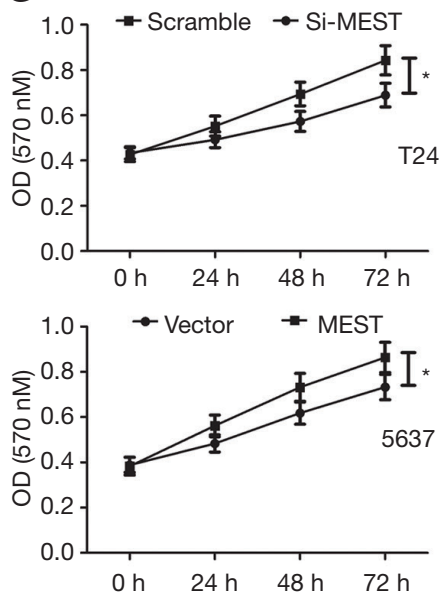

F
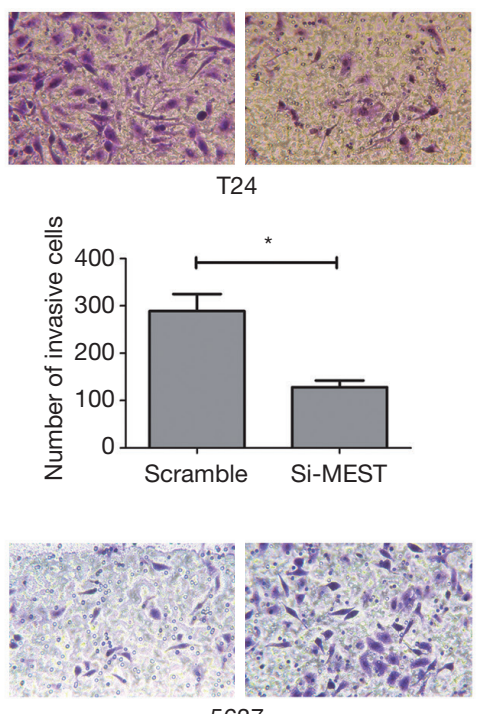

5637

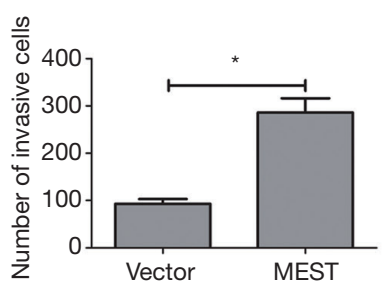

Figure 2 MEST promoted BC cell proliferation, migration, invasion. (A) Western blot detected the endogenous level of MEST in BC cell lines (5637, UM-UC-3, T24) and bladder epithelial immortalized cell SV-HUC-1; (B) Expression of MEST were detected by western blot when MEST knockdown or overexpression; MTT (C), clone formation (D), Wound scratch (magnification 100x) (E) and Transwell assays (magnification 200×) (F) were applied to assess the effects of MEST-depletion or overexpression- on BC cell proliferation, migration, invasion. ${ }^{*} \mathrm{P}<0.05,{ }^{* *} \mathrm{P}<0.01$. 
epithelial marker E-cadherin expression. Meanwhile, an opposite result was presented in MEST-overexpressed cells (Figure $3 A$ and $B$ ). In addition, immunofluorescence assay was further confirmed these result (Figure $3 C$ and $D$ ).

\section{MEST promotes BC cell proliferation, migration and invasion via STAT3/Twist-1}

A previous study showed that MEST plays an important role in STAT3 activation, which leads to increased Twist-1 expression, thereby triggering the EMT programme in breast cancer (15). Therefore, using western blot. we analysed the expression of STAT3/Twist-1 signalling components. As shown in Figure 4A, p-STAT3 and Twist-1 levels were repressed in MEST silenced cells, and their expressions were increased when MEST overexpression. But STAT3 level was not affected in MEST silenced or overexpressed cells. In additional, treating with STAT3 inhibitor S3I-201 could obviously attenuate STAT3 activation and Twist-1upregulation in MEST transfected 5637 cells. Moreover, STAT3 inhibitor could remarkably attenuated the oncogenic effect of MEST on proliferation, migration and invasion (Figure $4 B, C, D, E$ ).

\section{Discussion}

In this study, we confirmed that MEST was upregulated in $\mathrm{BC}$, especially in metastatic cancer tissues, compared with normal adjacent tissues. Moreover, MEST expression was obviously correlated with tumour stage, grade and lymph node metastasis. As an imprinted gene, MEST frequently undergoes LOI in a variety of cancers (8-13). Studies have confirmed that LOI has an effect on the expression of imprinted genes in human cancer; for example, IGF2 was reactivated and H19 was inactivated in Wilms' tumour and breast cancer, respectively, after LOI (16-18). Similarly, MEST is biallelic through promoter switching from isoform 1 to isoform $2(10,12,13,15)$. In terms of function, previous research has demonstrated that MEST can negatively regulate adipocyte differentiation and promote skeletal muscle growth and regeneration $(19,20)$. In cancer, MEST has been found to be overexpressed and to contribute to thyroid cancer cell survival, while MEST depletion has been demonstrated to lead to proliferation of inhibition and cell cycle arrest (8). Moreover, MEST was confirmed to promote breast cancer cell proliferation and metastasis $(15,21)$. Here, we also found that MEST facilitated cell proliferation, migration and invasiveness of $\mathrm{BC}$ cells.
However, in ovarian cancer, MEST was demonstrated to suppress cell proliferation, migration, invasion, tube formation, angiogenesis and tumorigenic ability (14). The reason MEST exerts opposite functions in different cancers may be attributed to different tumour characteristics and different binding targets.

Epithelial-mesenchymal transition (EMT) is a pivotal physiological process that is active during embryonic development, during which it functions in the formation of various germ layers and organs (22). During this process, epithelial cells lose polarity and expression of adhesion proteins, and acquire a mesenchymal phenotype and motility. An increasing number of studies has demonstrated that EMT is closely related to tumour invasion and metastasis (23-25). At the molecular level, the main features of EMT are characterised by the reduced expression of the epithelial marker E-cadherin and the enhanced expression of mesenchymal markers such as $\mathrm{N}$-cadherin and Vimentin, which implies tumour progression to a metastatic phenotype. E-cadherin belongs to a class of transmembrane glycoproteins that regulates intercellular homogeneity adhesion, while $\mathrm{N}$-cadherin is the principal component of epithelial structures, and thus, intercellular adhesion decreases with reduced E-cadherin levels and increased $\mathrm{N}$-cadherin expression, which results in tumour cell proliferation and metastasis (26). Vimentin is a type III intermediate filament that promotes tumour EMT phenotypes by reorganising the cytoskeleton to increase cell polarity and motility as well as generate cellular tension (27). In our study, we observed that MEST knockdown upregulated E-cadherin expression, whereas $\mathrm{N}$-cadherin and Vimentin levels were reduced. In addition, these results were completely reversed when MEST was overexpressed. Taken together, our data suggest that MEST can induce EMT in BC, which is consistent with previous studies in breast cancer (15).

Signal transducer and activator of transcription 3 (STAT3) belongs to the STAT family and is widely known as a facilitator of cell proliferation, survival, invasion, angiogenesis and immune evasion in cancer (28). When activated by the IL-6 family of cytokines, receptor tyrosine kinases or JAKs, STAT3 is primarily phosphorylated at the Y705 site, after which it is transported to the nucleus where it acts as a transcription factor with oncogenic functions (29). STAT3 plays a crucial role in tumorigenesis and progression, but whether aberrant STAT3 signalling also contributes to EMT, the early step of tumour invasion and metastasis, is still unknown. Previous studies have demonstrated 
A
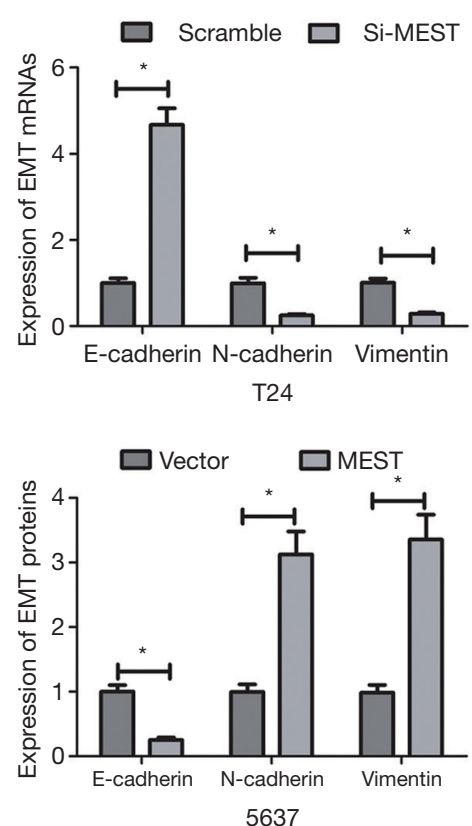

C T24
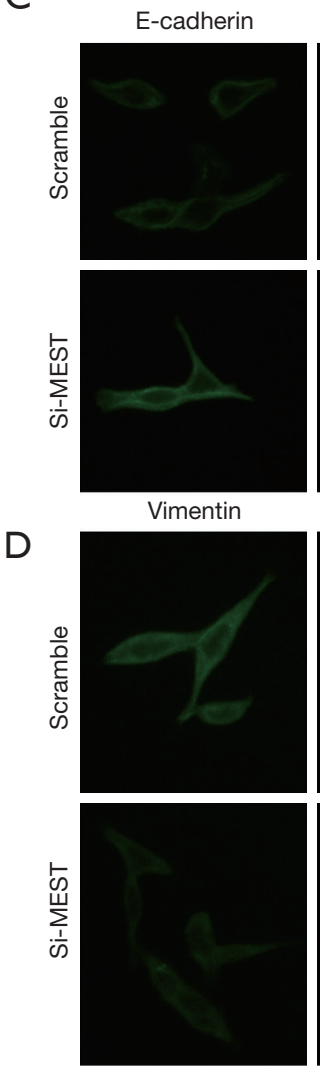

DAPI

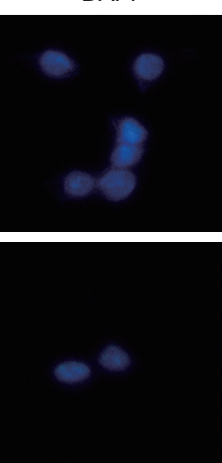

DAPI
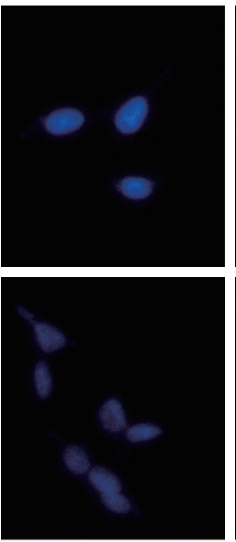

B

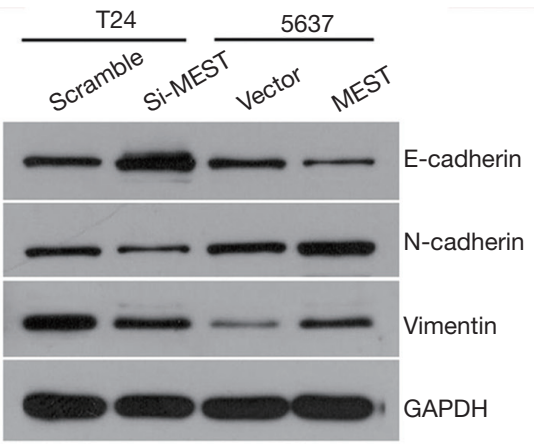

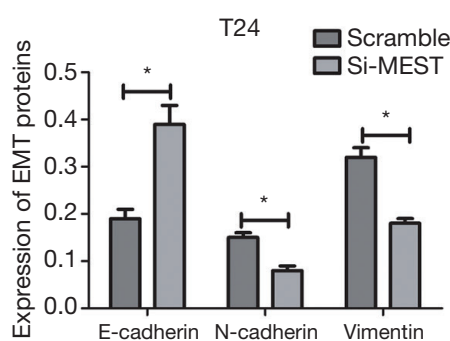

5637

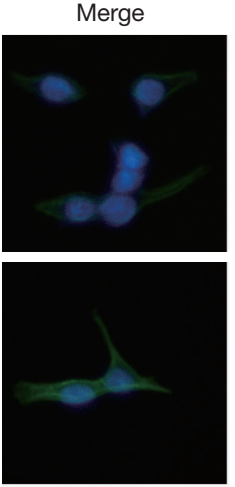

Merge
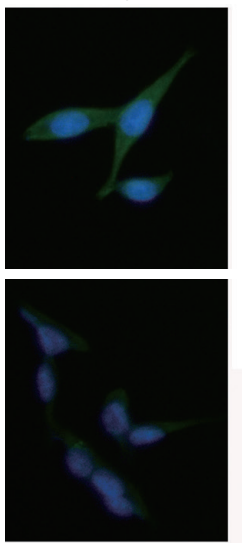

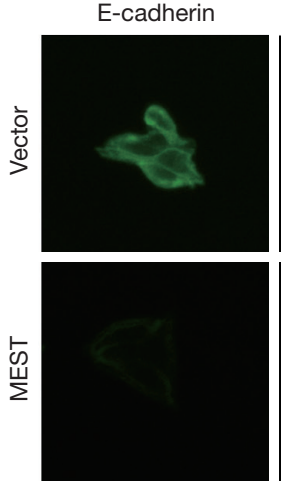

Vimentin
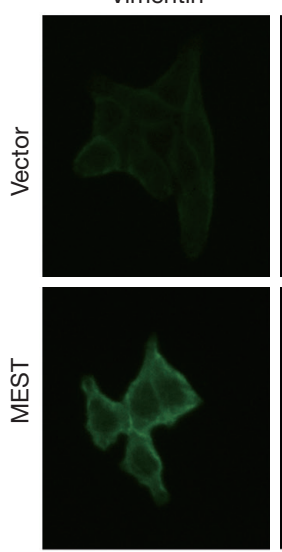

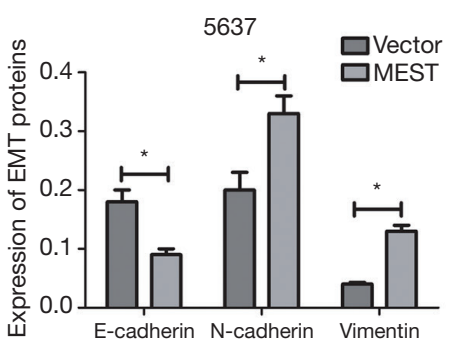

DAPI

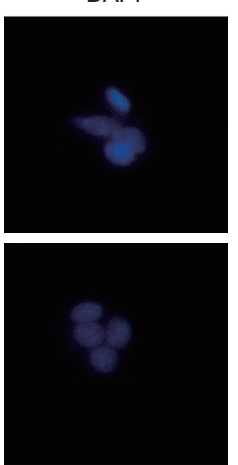

DAPI
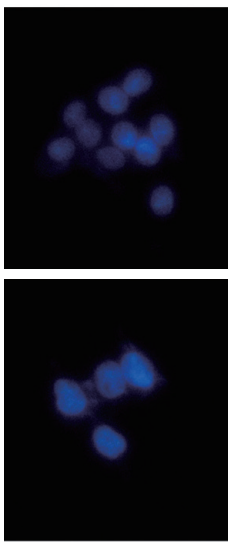

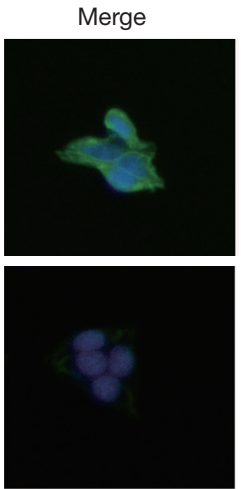

Merge

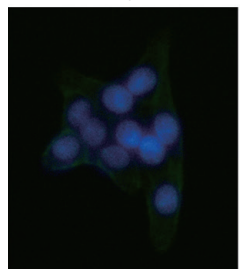

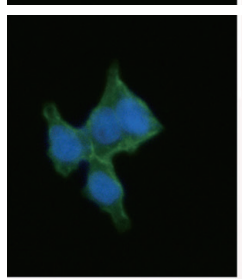

Figure 3 MEST induced epithelial-mesenchymal transition (EMT) in BC. qRT-PCR (A) and western blot (B) detected the expression of EMT markers including E-cadherin, N-cadherin and Vimentin in MEST-depletion or overexpression cells; Immunofluorescence analyzed the expression of EMT markers E-cadherin (C) and vimentin (D) (magnification 200x). The green signal represented the staining of corresponding protein, and the blue signal represented the nuclear staining by DAPI. ${ }^{*} \mathrm{P}<0.05$. 
A

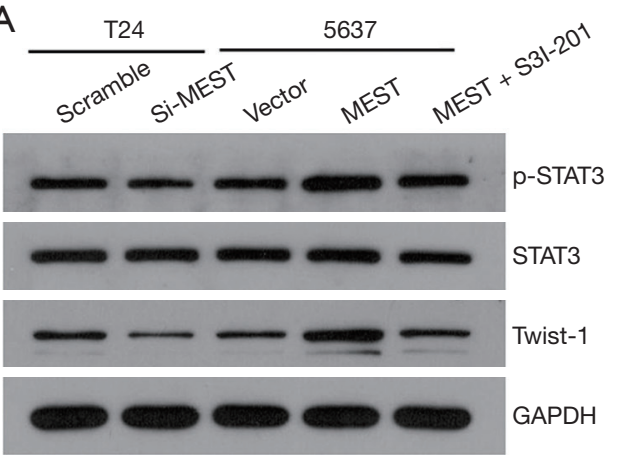

C
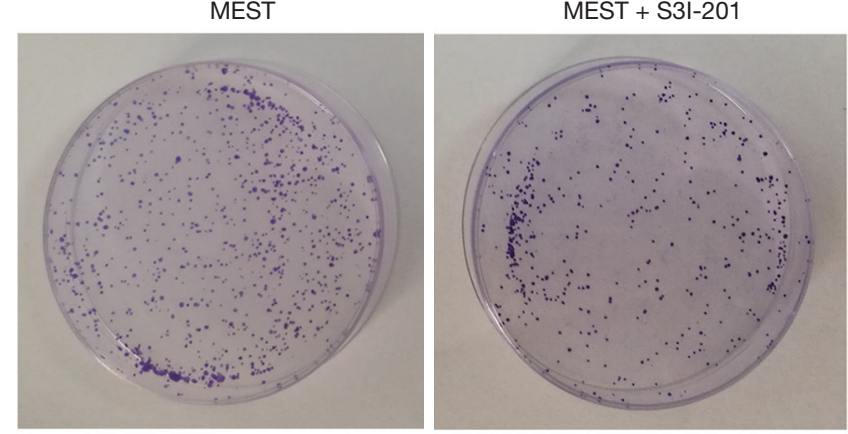

D
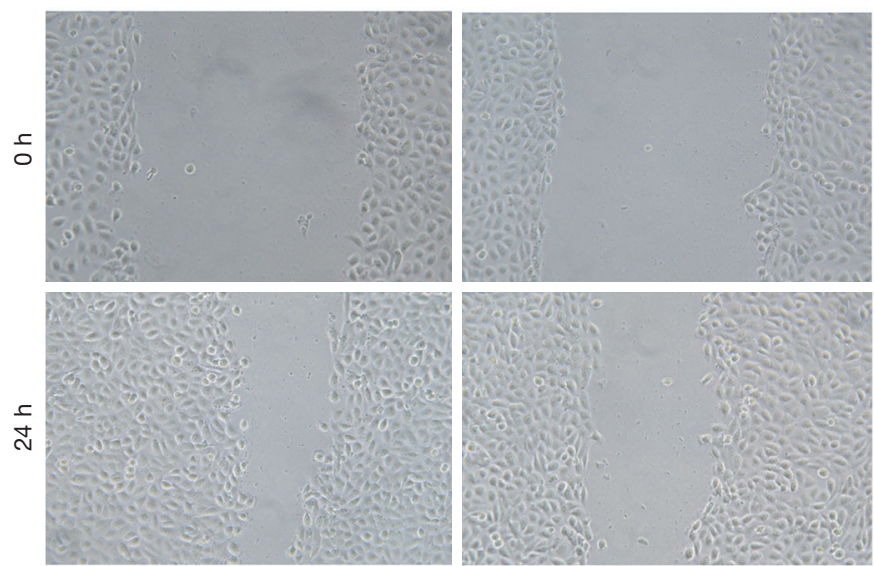

$\mathrm{E}$

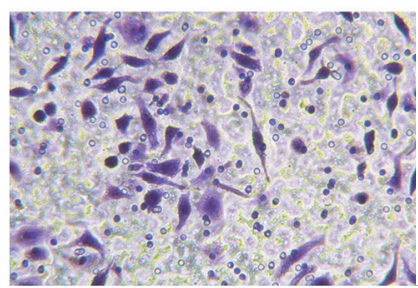

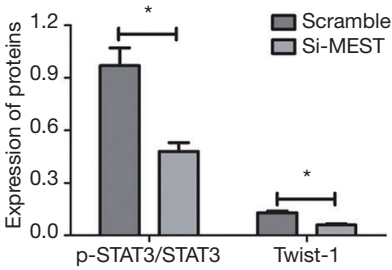

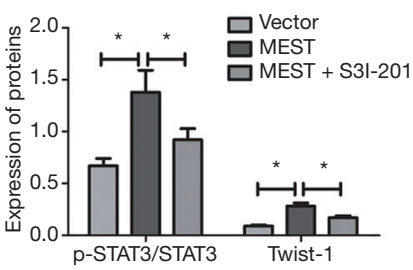

B

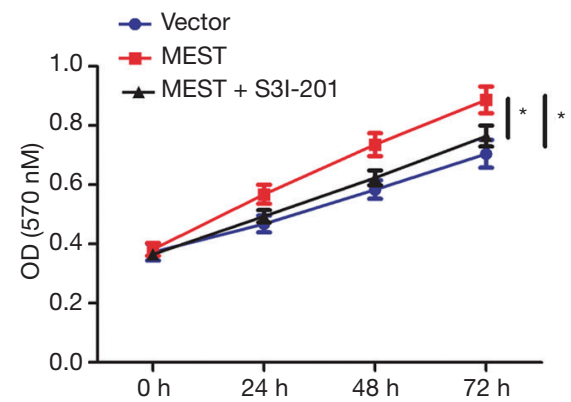

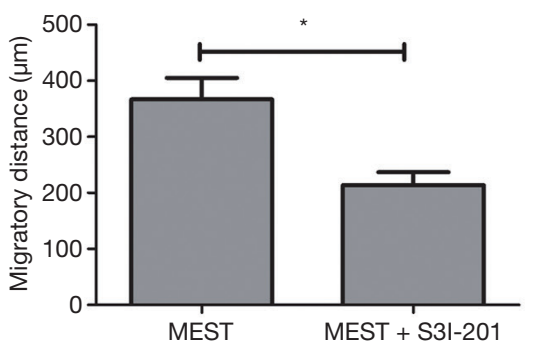

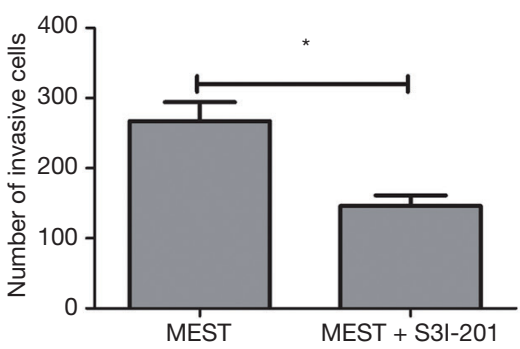

Figure 4 MEST promotes BC cell proliferation, migration and invasion via STAT3/Twist-1. (A) Western blot detected the expression of p-STAT3, STAT3 and Twist-1 in MEST-depletion or overexpression cells; Treating MEST transfected 5637 cells with STAT3 inhibitor S3I-201 (100 nM) for $24 \mathrm{~h}$, the cell proliferation, migration and invasion were detected by MTT (B), clone formation (C), Wound scratch (magnification 100×) (D) and Transwell assays (magnification 200x) (E), respectively. ${ }^{*} \mathrm{P}<0.05$. 
that STAT3 signalling contributes to EMT in cancer, including BC (30-32). STAT3 activation was shown to induce typical EMT-related morphological changes, which are accompanied by enhanced invasiveness and decreased expression of E-cadherin, while inhibition of STAT3 signalling impaired these effects (33). The regulatory mechanism that has been ascribed to STAT3 could regulate a series of EMT transcription factors such as Snail (34), Twist-1 (32) and ZEB1 (35). Recently, Kim et al. (15) confirmed that MEST-induced activated STAT3 was able to upregulate Twist-1 expression, which subsequently enabled the induction of EMT activation in breast cancer. Here, we also observed that MEST promoted p-STAT3 and Twist-1 expression, while treatment with a STAT3 inhibitor obviously attenuated STAT3 activation and Twist-1 upregulation induced by MEST. Moreover, inhibition of STAT3 signalling remarkably relieved the stimulating effects on BC cell proliferation, migration and invasion induced by MEST.

In summary, this study revealed that MEST promotes BC cell proliferation, migration and invasion via STAT3/Twist1-mediated EMT. It is therefore suggested that MEST may represent a promising target for improving BC therapy.

\section{Acknowledgments}

Funding: None.

\section{Footnote}

Reporting Checklist: The authors have completed the MDAR checklist. Available at http://dx.doi.org/10.21037/tcr-201006

Data Sharing Statement: Available at http://dx.doi. org/10.21037/tcr-20-1006

Conflicts of Interest: All authors have completed the ICMJE uniform disclosure form (available at http://dx.doi. org/10.21037/tcr-20-1006). The authors have no conflicts of interest to declare.

Ethical Statement: The authors are accountable for all aspects of the work in ensuring that questions related to the accuracy or integrity of any part of the work are appropriately investigated and resolved. The study was conducted in accordance with the Declaration of Helsinki (as revised in 2013). The study was approved by the institutional ethics committee of Xiangya Hospital, Central South University (approval No. 2019012618), and written informed consents were obtained from all patients.

Open Access Statement: This is an Open Access article distributed in accordance with the Creative Commons Attribution-NonCommercial-NoDerivs 4.0 International License (CC BY-NC-ND 4.0), which permits the noncommercial replication and distribution of the article with the strict proviso that no changes or edits are made and the original work is properly cited (including links to both the formal publication through the relevant DOI and the license). See: https://creativecommons.org/licenses/by-nc-nd/4.0/.

\section{References}

1. Mahdavifar N, Ghoncheh M, Pakzad R, et al. Epidemiology, Incidence and Mortality of Bladder Cancer and their Relationship with the Development Index in the World. Asian Pac J Cancer Prev 2016;17:381-6.

2. Terracciano D, Ferro M, Terreri S, et al. Urinary long noncoding RNAs in nonmuscle-invasive bladder cancer: new architects in cancer prognostic biomarkers. Transl Res 2017;184:108-17.

3. Tian DW, Wu ZL, Jiang LM, et al. KIF5A Promotes Bladder Cancer Proliferation In Vitro and In Vivo. Dis Markers 2019;2019:4824902.

4. Yang C, Zhang W, Wang L, et al. Musashi-2 promotes migration and invasion in bladder cancer via activation of the JAK2/STAT3 pathway. Lab Invest 2016;96:950-8.

5. Pedersen IS, Dervan P, McGoldrick A, et al. Promoter switch: a novel mechanism causing biallelic PEG1/MEST expression in invasive breast cancer. Hum Mol Genet 2002;11:1449-53.

6. Lefebvre L, Viville S, Barton SC, et al. Genomic structure and parent-of-origin-specific methylation of Peg1. Hum Mol Genet 1997;6:1907-15.

7. Mayer W, Hemberger M, Frank HG, et al. Expression of the imprinted genes MEST/Mest in human and murine placenta suggests a role in angiogenesis. Dev Dyn 2000;217:1-10.

8. Boot A, Oosting J, de Miranda NF, et al. Imprinted survival genes preclude loss of heterozygosity of chromosome 7 in cancer cells. J Pathol 2016;240:72-83.

9. Kohda M, Hoshiya H, Katoh M, et al. Frequent loss of imprinting of IGF2 and MEST in lung adenocarcinoma. Mol Carcinog 2001;31:184-91.

10. Nakanishi H, Suda T, Katoh M, et al. Loss of imprinting of PEG1/MEST in lung cancer cell lines. Oncol Rep 
2004;12:1273-8.

11. Pedersen IS, Dervan PA, Broderick D, et al. Frequent loss of imprinting of PEG1/MEST in invasive breast cancer. Cancer Res 1999;59:5449-51.

12. Moon YS, Park SK, Kim HT, et al. Imprinting and expression status of isoforms 1 and 2 of PEG1/MEST gene in uterine leiomyoma. Gynecol Obstet Invest 2010;70:120-5.

13. Nishihara S, Hayashida T, Mitsuya K, et al. Multipoint imprinting analysis in sporadic colorectal cancers with and without microsatellite instability. Int J Oncol 2000;17:317-22.

14. Ruan Z, Zhao D. Long intergenic noncoding RNA LINC00284 knockdown reduces angiogenesis in ovarian cancer cells via up-regulation of MEST through NFkappaB1. FASEB J 2019;33:12047-59.

15. Kim MS, Lee HS, Kim YJ, et al. MEST induces Twist1-mediated EMT through STAT3 activation in breast cancers. Cell Death Differ 2019;26:2594-606.

16. Ogawa O, Eccles MR, Szeto J, et al. Relaxation of insulinlike growth factor II gene imprinting implicated in Wilms' tumour. Nature 1993;362:749-51.

17. Rainier S, Johnson LA, Dobry CJ, et al. Relaxation of imprinted genes in human cancer. Nature 1993;362:747-9.

18. Yballe CM, Vu TH, Hoffman AR. Imprinting and expression of insulin-like growth factor-II and H19 in normal breast tissue and breast tumor. J Clin Endocrinol Metab 1996;81:1607-12.

19. Hiramuki Y, Sato T, Furuta Y, et al. Mest but Not MiR335 Affects Skeletal Muscle Growth and Regeneration. PLoS One 2015;10:e0130436.

20. Karbiener M, Glantschnig C, Pisani DF, et al. Mesodermspecific transcript (MEST) is a negative regulator of human adipocyte differentiation. Int J Obes (Lond) 2015;39:1733-41.

21. Chen L, Wu X, Xie H, et al. ZFP57 suppress proliferation of breast cancer cells through down-regulation of MESTmediated Wnt/beta-catenin signalling pathway. Cell Death Dis 2019;10:169.

22. Zhou Q, Chen S, Lu M, et al. EFEMP2 suppresses epithelial-mesenchymal transition via Wnt/beta-catenin signaling pathway in human bladder cancer. Int J Biol Sci 2019; 15:2139-55.

23. Ding NH, Zhang L, Xiao Z, et al. NEK4 kinase regulates EMT to promote lung cancer metastasis. J Cell Mol Med 2018;22:5877-87.

24. Liu L, Wu N, Wang Y, et al. TRPM7 promotes the epithelial-mesenchymal transition in ovarian cancer through the calcium-related PI3K / AKT oncogenic signaling. J Exp Clin Cancer Res 2019;38:106.

25. Zeng Z, Yang H, Xiao S. ACTL6A expression promotes invasion, metastasis and epithelial mesenchymal transition of colon cancer. BMC Cancer 2018;18:1020.

26. Tao Y, Zhao Z, Ma J, et al. MiR-214-3p regulates the viability, invasion, migration and EMT of TNBC cells by targeting ST6GAL1. Cytotechnology 2019;71:1155-65.

27. Liu CY, Lin HH, Tang MJ, et al. Vimentin contributes to epithelial-mesenchymal transition cancer cell mechanics by mediating cytoskeletal organization and focal adhesion maturation. Oncotarget 2015;6:15966-83.

28. Zheng M, Cao MX, Yu XH, et al. STAT3 Promotes Invasion and Aerobic Glycolysis of Human Oral Squamous Cell Carcinoma via Inhibiting FoxO1. Front Oncol 2019;9:1175.

29. Yu H, Lee H, Herrmann A, et al. Revisiting STAT3 signalling in cancer: new and unexpected biological functions. Nat Rev Cancer 2014;14:736-46.

30. Chen MJ, Deng J, Chen C, et al. LncRNA H19 promotes epithelial mesenchymal transition and metastasis of esophageal cancer via STAT3/EZH2 axis. Int J Biochem Cell Biol 2019;113:27-36.

31. Peng W, He D, Shan B, et al. LINC81507 act as a competing endogenous RNA of miR-199b-5p to facilitate NSCLC proliferation and metastasis via regulating the CAV1/STAT3 pathway. Cell Death Dis 2019;10:533.

32. Zhao D, Besser AH, Wander SA, et al. Cytoplasmic p27 promotes epithelial-mesenchymal transition and tumor metastasis via STAT3-mediated Twist1 upregulation. Oncogene 2015;34:5447-59.

33. Li B, Huang C. Regulation of EMT by STAT3 in gastrointestinal cancer (Review). Int J Oncol 2017;50:753-67.

34. Saitoh M, Endo K, Furuya S, et al. STAT3 integrates cooperative Ras and TGF-beta signals that induce Snail expression. Oncogene 2016;35:1049-57.

35. Avtanski DB, Nagalingam A, Bonner MY, et al. Honokiol inhibits epithelial-mesenchymal transition in breast cancer cells by targeting signal transducer and activator of transcription 3/Zeb1/E-cadherin axis. Mol Oncol 2014;8:565-80.

Cite this article as: Zhao $\mathrm{C}, \mathrm{Hu} \mathrm{X}$, Tong $\mathrm{S}, \mathrm{Mo} \mathrm{M}, \mathrm{He}$ W, Wang L, Li Y. MEST promotes bladder cancer cell proliferation, migration and invasion via STAT3/Twist-1mediated EMT. Transl Cancer Res 2020;9(10):6178-6188. doi $10.21037 /$ tcr-20-1006 